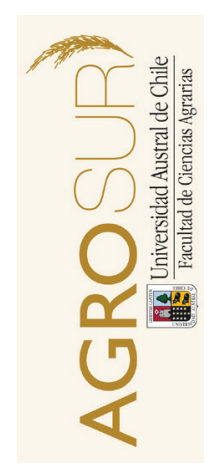

\title{
Evaluación del establecimiento de tres cultivares de achicoria (Cichorium intybus L.) en siembra asociada a ballica bianual y avena en la comuna de Purranque
}

\author{
Evaluation of the establishment of three cultivars of chicory \\ (Cichorium intybus L.) in association with biennial \\ ryegrass and oats in Purranque \\ Momberg, J. ${ }^{a}$, Balocchi, O.A. ${ }^{b}$ Keim, J.P. ${ }^{b *}$ \\ ${ }^{a}$ Escuela de Agronomía, Facultad de Ciencias Agrarias, Universidad Austral Chile. \\ ${ }^{b}$ Instituto de Producción Animal, Universidad Austral de Chile. Casilla 567, Valdivia, Chile.
}

\begin{tabular}{|c|}
\hline $\begin{array}{l}\text { Article history: } \\
\text { Received 06.03.2017 } \\
\text { Accepted 23.03.2018 }\end{array}$ \\
\hline $\begin{array}{l}\text { Keywords: } \\
\text { Supplementary crops } \\
\text { Chicory } 501 \\
\text { Punter }\end{array}$ \\
\hline $\begin{array}{l}\text { Original Research Article, } \\
\text { Animal Science }\end{array}$ \\
\hline $\begin{array}{l}\text { *Corresponding author: } \\
\text { J.P. Keim } \\
\text { E-mail address: } \\
\text { juan.keim@uach.cl }\end{array}$ \\
\hline
\end{tabular}

A B S T R A C T

Chichorium intybus L. is an emergent supplementary crop for farmers in southern Chile, and there is little knowledge about its management and production. The aim of this study was to evaluate the establishment, production, nutritional quality and morphological components of different chicory varieties (Chicory 501, Choice and Punter) sown in mixture with Lolium multiflorum Lam. and oats. Germination, botanical composition and nutritional quality were measured. To analise the establishment and botanical composition, a completely random design was used in an area of 4.5 hectares, divided into nine plots with three replicates for each cultivar. For the analysis of yield and morphological composition, Anova was performed. No significant differences were observed between the three cultivars at establishment (first three cuts) for DM yield, but in the last cut Punter achieved the highest yield, followed by Choice and finally Chicory 501 (106.1, 40.5 and 6, 9 kg DM $\mathrm{ha}^{-1}$, respectively). According to the botanical composition, the species with the highest contribution was L. multiflorum ( $98 \%$ accumulated). The morphological characteristics showed that Choice presented larger leaves (13.38 cm long), Chicory 501 had the higher amount of leaves (2.98) and Punter registered the greatest leaf/stem ratio (2.78).

\section{RESUMEN}

En Chile, Cichorium intybus L. (achicoria) es un cultivo incipiente, existen pocos cultivares comercializados y poco conocimiento sobre su manejo y producción. El objetivo fue evaluar el establecimiento, producción, calidad nutritiva y componentes morfológicos de variedades de achicoria (Chicory 501, Choice y Punter) en mezcla con ballica bianual y avena. Se determinó germinación, composición botánica y calidad nutricional. Para el establecimiento y composición botánica se utilizó un diseño completamente al azar en un área de 4,5 hectáreas, dividida en nueve parcelas con tres repeticiones para cada cultivar. Se establecieron cinco fechas de corte para medir crecimiento de los cultivares y su porcentaje en relación con las otras especies. Para el análisis de producción y composición morfológica, se utilizó Andeva y test de Tukey. El poder germinativo mostró ser bajo, asociado al tamaño de semillas de achicoria. El establecimiento no mostró diferencias significativas en las producciones de los tres cultivares en los tres primeros cortes, pero sí en los últimos, donde Punter logra la mayor producción, seguido por Choice y finalmente Chicory 501 (106,1, 40,5 y 6,9 kg MS ha-1, respectivamente). La composición botánica muestra que la especie con mayor contribución es ballica (98\% acumulado). Las características morfológicas mostraron que Choice presentó hojas más grandes (13,38 cm de largo), Chicory 501 mayor cantidad de hojas (2,98) y Punter la mejor relación hoja/tallo (2,78).

Palabras clave: Cultivos suplementarios, Chicory 501, Punter.

\section{INTRODUCCIÓN}

Las praderas del sur de Chile están compuestas principalmente por especies pertenecientes a la clase de las angiospermas, siendo las principales las gramíneas de la subclase monocotiledóneas y las leguminosas de la subclase de las dicotiledóneas (Aedo, 1996). La distribución de crecimiento de la pradera varía según la épo- ca del año, observándose mayor crecimiento en primavera y otoño en comparación a invierno y verano (Ruiz, 1996). La calidad nutritiva de las praderas también cambia dependiendo de la época del año, probablemente por efecto del aumento de la temperatura y horas de sol, dado que ambas variables producen un aumento en los carbohidratos estructurales y una disminución de los carbohidratos no estructurales (Ruiz, 1996). 
Ballica italiana (L. multiflorum) posee un ciclo de vida más corto y un tamaño más erecto que la ballica perenne (Lolium perenne L.), con macollos de mayor tamaño y hojas más anchas. La raíz es similar a la ballica perenne. La inflorescencia de ballica italiana, la cual puede ser anual o bianual, posee número mayor de inflorescencias que ballica perenne (Ruiz, 1996).

La ballica italiana como gramínea puede prosperar en una gran gama de suelos, siendo menos exigente en este sentido que las leguminosas, pero se recomienda su siembra en otoño (López, 1996). Se caracteriza por tener un amplio rango de época de siembra, siendo de rápido establecimiento y de satisfactorio crecimiento en otoño y primavera (Ruiz, 1996). La calidad nutritiva varía según la estación del año presentando en invierno y primavera porcentajes de materia seca, proteína cruda y energía metabolizable similares, con un 13\%, 23\% y 2,8 Mcal kg MS ${ }^{-1}$, respectivamente (Ruiz, 1996).

Estero (2013) menciona que la achicoria forrajera es una planta originaria de la zona del mediterráneo, de la familia de las Asteráceas. Para obtener buenos resultados productivos se recomienda su siembra en mezcla con trébol blanco (Trifolium repens L.) por su alta demanda de nitrógeno (Bavera, 2009).

Es una planta herbácea que tiene crecimiento erecto, con una raíz pivotante y gruesa, de ciclo bianual e incluso, perenne. Las hojas basales son aserradas y crecen en forma de roseta, las hojas de la parte superior son alternas, pequeñas y sus dientes son menores. Los tallos son largos, con látex e irregularmente ramificados. Produce gran cantidad de flores de color azul claro, de hasta $4 \mathrm{~cm}$ de diámetro (Estero, 2013).

C. intybus es una forrajera de alto valor proteico, rústica y una vez desarrollada compite muy bien con las malezas. Es una planta muy exigente de nitrógeno y por ende requiere suelos de mediana a buena fertilidad (se siembra en surcos o al voleo, con dosis por hectárea cercana a los $6 \mathrm{~kg}$ si se siembra sola, y entre 2 a $4 \mathrm{~kg} \mathrm{ha}^{-1}$ si se siembra en mezcla con otras especies) (Li y Kemp, 2005). La profundidad de siembra no debe ser mayor de $0,5 \mathrm{~cm}$ puesto que es una semilla muy pequeña y a una mayor profundidad tendrá dificultades para emerger (Bavera, 2009). Dependiendo de la fecha de siembra será la época de utilización de la pradera, siendo recomendable su siembra desde febrero a octubre, pero mientras antes se siembre, mejor tolerará el invierno y tendrá una mayor producción en primavera (Bavera, 2009).

Las características que se han observado en otros países de clima mediterráneo en el primer año son, un muy rápido establecimiento en relación con otras especies, con excelente adaptación al pastoreo, gran respuesta a la fertilización nitrogenada, con una elevada producción de forraje en primavera y verano (sobre los $100 \mathrm{~kg} \mathrm{MS} \mathrm{ha}^{-1} \mathrm{~d}^{-1}$ ) (Li y Kemp, 2005). Las características nutritivas de la achicoria muestran que tiene cerca de un $20 \%$ de proteína cruda, una fibra detergente ácido cercano al 23\%, una fibra detergente neutro de alrededor de un $26 \%$, azúcar soluble de un $13 \%$, almidón cercano a 1,4\%, $14 \%$ de cenizas totales, una digestibilidad in vitro de la MS del 73\% y una energía metabolizable cercana a 2,8 $\mathrm{Mcal} \mathrm{kg} \mathrm{MS}^{-1}$, todo esto en base a materia seca (Glassey, 2013).

Esta planta debe ser defoliada con un mínimo de 7 hojas y cuando tiene una altura entre 25 y $35 \mathrm{~cm}$, esto porque su sistema radicular profundo necesita que la planta le entregue suficiente energía para seguir creciendo y así aumentar su potencial de crecimiento (Bavera, 2009) y permite un balance entre persistencia de la pradera y mantener una calidad nutricional adecuada (Lee et al., 2015a).

Deak et al. (2007) indican que a medida que aumenta el número de especies en la pradera, su contribución disminuye paulatinamente. Rumball (1986) menciona que esto ocurre por dos razones, una es que los animales la prefieren frente al resto de pasturas, por lo que la consumen más y la otra es que no encuentra espacios en la pradera por no ser buena competidora. En un estudio realizado en el noreste de Estados Unidos se observó que la achicoria, variedad Puna, disminuye su participación dentro de la pradera (18,1\% a un $7,3 \%$ de contribución), demostrando que no es buena competidora (Deak et al., 2007).

En Chile la achicoria es un cultivo incipiente, por lo que existen pocos cultivares comercializados a nivel nacional, además del poco conocimiento por parte de los agricultores y las empresas importadoras sobre su manejo y producción. Es por esto que resulta interesante una comparación entre las variedades presentes en el país para ver su comportamiento en mezcla.

Chicory 501 es un cultivar reciente que se caracteriza por entregar elevados rendimientos de materia seca, con hábito de crecimiento erecto y con su punto de crecimiento muy bajo (AGRISEEDS, 2014). Choice fue creada con el propósito de ser usada para la alimentación animal, por lo que en Australia ha sido utilizada con ovinos y con vacunos de leche y carne. Además, tiene alta productividad y buena calidad nutritiva en la época estival (AGRICOM, 2012). SF Punter es una achicoria perenne de alto rendimiento, con gran vigor al establecimiento y de buena producción en verano e invierno, fue probada al igual que Chicory 501 en Nueva Zelanda y provee de buenas características nutritivas, principalmente energéticas, por lo que aumenta la producción de los animales (Seed Force, 2009).

El objetivo general de este estudio fue evaluar el establecimiento, producción, calidad nutritiva y componentes morfológicos de tres variedades de achicoria forrajera en mezcla con ballica bianual y avena. 


\section{MATERIAL Y METODO}

El estudio se realizó en la Décima Región de Chile, provincia de Osorno, comuna de Purranque. Según el diseño, completamente al azar, en un área total de 4,5 hectáreas se distribuyeron nueve parcelas de 0,5 hectáreas, donde se designaron tres repeticiones para cada cultivar. La fecha de siembra fue el día 14 de marzo de 2013, con dosis comerciales de mezclas forrajeras: $3 \mathrm{~kg}$ $\mathrm{ha}^{-1}$ de cada cultivar de achicoria, $50 \mathrm{~kg} \mathrm{ha}^{-1}$ de ballica bianual cultivar Delish y $50 \mathrm{~kg} \mathrm{ha}^{-1}$ de avena cultivar Urano. Las nueve parcelas se repartieron al azar, conformando tres grupos, cada cual fue sembrado con un cultivar escogido para este estudio: Chicory 501, Choice y Punter.

El lugar en el cual se estableció la pradera fue anteriormente sembrado con raps el año 2012, el cual se cosechó en enero del 2013. Posteriormente, se aplicaron $1000 \mathrm{~kg} \mathrm{CaCO}_{3} \mathrm{ha}^{-1}$. La preparación del suelo consistió en dos rastrajes y una preparación final de la cama de semilla con vibro cultivador. La siembra se realizó con SEMEATO modelo TDAX 3500. Las semillas de ballica y avena se mezclaron y se colocaron en el cajón de la semilla mientras que la achicoria se sembró con el compartimiento destinado al trébol. La fertilización se realizó al momento de la siembra con una dosis de $250 \mathrm{~kg}$ ha $^{-1}$ de Microessentials Zinc (N: 11\%, $\mathrm{P}_{2} \mathrm{O}_{5}: 48 \%$, S: 5\% y $\mathrm{Zn}: 0,6 \%$ ). Posterior a la siembra se utilizaron rollos compactadores, para favorecer el contacto de la semilla con el suelo y evitar mayores pérdidas de evapotranspiración. Posterior a la siembra, se fertilizó dos veces con urea (46\% N). El día 18 de abril se aplicaron $100 \mathrm{~kg}$ por hectárea al voleo con trompo y el día 28 de agosto se aplicaron $150 \mathrm{~kg} \mathrm{ha}^{-1}$, totalizando $250 \mathrm{~kg} \mathrm{ha}^{-1}$ de urea.

\section{Porcentaje de germinación de semillas}

Para evaluar y obtener el porcentaje de germinación de cada cultivar de $C$. intybus en estudio, se pesaron 500 semillas de cada cultivar en balanza electrónica de precisión de $1000 \mathrm{~g}$.

Para obtener el número de plantas emergidas (\% de germinación) de los distintos cultivares, se procedió a dividir en cinco repeticiones de 100 semillas de cada cv. en placas petri con papel absorbente humedecido para favorecer su hidratación. Las placas petri, se procedieron a colocar en una base metálica, con flujo de agua que permitió humedecimiento del papel conectado a la placa, todo esto para evitar deshidratación de las semillas y homologar la situación de germinación en suelo a capacidad de campo. La duración de este procedimiento fue de quince días corridos en el laboratorio de producción animal a temperatura ambiente. Al cabo de los quince días, se evaluó en cada placa la cantidad de semillas germinadas, logrando obtener así el porcentaje de germinación.

\section{Producción de MS y composición botánica}

En cada una de las parcelas se instaló una jaula de exclusión de un metro cuadrado después de la siembra, para evaluar el crecimiento de las especies en mezcla, sin perturbaciones derivadas de los animales que pastorearon el resto de la parcela. Estas jaulas fueron hechas de madera de pino de una altura de $40 \mathrm{~cm}$ rodeadas y cubiertas con malla para impedir que los animales coman o destruyan el interior.

Se realizaron cinco cortes de la superficie excluida: el 4 de mayo, 23 de junio, 31 de agosto, 22 de octubre y 26 de noviembre de 2013.

\section{Producción por mezcla forrajera}

En cada fecha de corte de cada jaula de exclusión, se procedió a tomar dos submuestras de $100 \mathrm{~g}$ cada una, una de las cuales se utilizó para la obtención de materia seca total y la otra para su análisis botánico. Los cortes se realizaron cada vez que la pradera alcanzó una altura cercana a los $20 \mathrm{~cm}$, cortándose a los $5 \mathrm{~cm}$ de altura para obtener un buen rebrote de la pradera y simulando un pastoreo rotativo. Las jaulas de exclusión permanecieron siempre en el mismo lugar.

Primero se pesó la materia verde en balanza digital y posteriormente se procedió a secar en horno en laboratorio por 48 horas. Posteriormente se extrae la muestra del horno, se pesa y con este valor se obtiene el porcentaje de materia seca en relación con peso inicial de la submuestra en verde.

\section{Composición botánica y producción por especies}

Posteriormente, en la segunda submuestra de la materia verde segada en cada fecha del experimento, se separaron en forma manual las especies. Éstas fueron secadas cada una por separado en un horno de aire forzado a una temperatura de $60{ }^{\circ} \mathrm{C}$ durante 48 horas o hasta que el peso se hizo constante, obteniéndose así el peso seco por especie y el porcentaje relativo de contribución de cada una de éstas al peso total de la muestra. El rendimiento por especies se obtuvo al sumar las producciones acumuladas de cada especie. Estas se determinaron al calcular el crecimiento en un período (entre dos pastoreos), multiplicado por la contribución de cada especie, expresada en porcentaje, por la producción por corte de cada mezcla forrajera.

\section{Componentes del rendimiento}

Antes de la entrada de los animales al potrero se instalaron los cinco cuadrantes de $400 \mathrm{~cm}^{2}$ al azar para obtener 10 plantas del interior de cada uno de ellos. A estas plantas de achicoria se les contó el número de hojas, se midió el largo de éstas con una regla y se obtuvo 
el contenido de materia seca de cada una de las estructuras, separándolas en el laboratorio de forrajeras de la Universidad Austral de Chile para lograr la relación hoja/peciolo. Después de esto, se dejaron secando en hornos de $60^{\circ} \mathrm{C}$ por 48 horas.

El largo de las hojas se midió con una regla, los peciolos se separaron de las hojas cortándolos con una tijera y, después, fueron pesados en verde y en seco con una balanza electrónica.

Para determinar las características morfológicas de las especies pratenses se efectuó un muestreo al azar con el método del cuadrante $(20 \times 20 \mathrm{~cm})$ cinco veces en cada parcela, segando 10 plantas de las especies en mezcla de siembra a evaluar en el experimento. Con esto además se logró determinar los componentes del rendimiento, puesto que se obtuvieron los pesos secos de las distintas estructuras de las plantas y con esto el peso seco promedio de cada una de las plantas, además de saber cómo se distribuye éste dentro de ellas.

Para obtener los datos de relación hoja/peciolo, se dividió el peso de las hojas de una variedad por el peso de los peciolos de la misma variedad.

\section{Composición nutricional}

Con el forraje cosechado después del cuarto corte se hizo un análisis nutricional en el laboratorio de $\mathrm{Nu}$ trición Animal de la Universidad Austral de Chile para determinar las diferencias y/o similitudes entre los distintos cultivares de achicoria forrajera. Para poder realizar este análisis se mezclaron las tres repeticiones de cada cultivar en estudio.

\section{Diseño Experimental y Análisis Estadístico}

El diseño de experimentos consistió en un diseño completamente al azar, en un área definida de 4,5 hectáreas, subdividida para efectos del presente estudio en nueve parcelas de 0,5 hectáreas, las cuales fueron seleccionadas al azar para la siembra en mezcla de las especies forrajeras con un cultivar de $C$. intybus, estableciéndose para ello tres parcelas o repeticiones para cada variedad o cultivar.

Con los datos obtenidos, para poder analizar y comparar el comportamiento de los cultivares en estudio de achicoria forrajera, se realizó un análisis de varianza con medidas repetidas en el tiempo con un nivel de significancia del 5\%, donde el cultivar de achicoria correspondió al factor principal y la época de muestreo a la medida repetida en el tiempo. Cuando el valor-p fue menor que 0,05 , se realizó el test de comparación de medias de Tukey. Para el caso de las variables relacionadas con composición botánica y concentración nutricional, sólo se reportan los valores promedio, ya que la cantidad de achicoria obtenida en cada unidad experimental fue muy baja y se debió hacer una muestra compuesta.

\section{RESULTADOS Y DISCUSIÓN}

\section{Porcentaje de germinación de Cultivares}

En lo que respecta a las semillas, el cultivar que presentó un menor peso en 500 semillas fue Punter (0,673 g), seguido de Chicory 501 (0,692 g) y Choice $(0,694 \mathrm{~g})$. Las diferencias son mínimas y el peso extremadamente liviano. A los quince días del ensayo de germinación, el cultivar con mayor porcentaje fue Choice seguido de Punter y el menor porcentaje germinativo fue Chicory 501 (63, 57 y $55 \%$, respectivamente).

Balocchi $(2013)^{1}$, indica que el mínimo exigido para la gran mayoria de las semillas en la prueba de germinación es de un $80 \%$ y en este caso se obtuvo un $60 \%$, lo que disminuye de gran forma el número de plantas por hectárea, estos valores se encuentran por debajo del $97 \%$ reportado por Reed et al. (2008) para C. intybus var. Grouse en el sur de Australia.

\section{Rendimiento y Composición botánica}

\section{Composición botánica}

Al evaluar la contribución de la achicoria en la composición botánica, obtenemos que es muy deficiente y que además disminuyó a medida que avanzaba el número de cortes. En el corte 1 el porcentaje promedio de las 3 variedades fue un poco superior a un $3 \%$, disminuyendo en el segundo corte a un 2,5\% y terminando con el quinto corte con solo un 1,6\%. Al revisar estos datos, pero por variedad, obtenemos que la de mayor importancia en el cultivo fue Punter, con casi un 2,7\% de composición botánica promedio de todos los cortes. Choice y Chicory 501 fueron muy similares y obtuvieron apenas un 1,6\%, siendo levemente superior Chicory 501.

De acuerdo con la Figura 1, en la primera fecha de corte, es decir en mayo, ballica es la que posee el mayor porcentaje con un $74 \%$ seguido de avena con un $23 \%$ donde la especie achicoria solo aporta un 3\%. En junio, es decir al realizarse el segundo corte, la ballica continúa siendo la especie con mayor porcentaje de prevalencia en la composición, aumentando de un $74 \%$ a un $79 \%$, disminuyendo el porcentaje de avena de un 23\% a un $18 \%$, manteniéndose achicoria en un 3\%. En el tercer corte, la ballica aumenta de un $79 \%$ a un $96 \%$, pero la avena disminuye de un $18 \%$ a solo un $2 \%$ y la achicoria aporta únicamente un 1\%. Esta distribución, en donde la ballica representa un $96 \%$ de las especies y tan solo un $1 \%$ de achicoria, se debería entre otras causas a la mayor agresividad al establecimiento de las gramíneas de ciclo anual y a los cortes que afectan el crecimiento de los cv de achicoria. Ya en el cuarto corte, la ballica au-

Balocchi, O., 2013. Ing. Agr., M. Sc., Ph. D. Profesor titular Instituto de Producción Animal, Universidad Austral de Chile. Comunicación personal. 


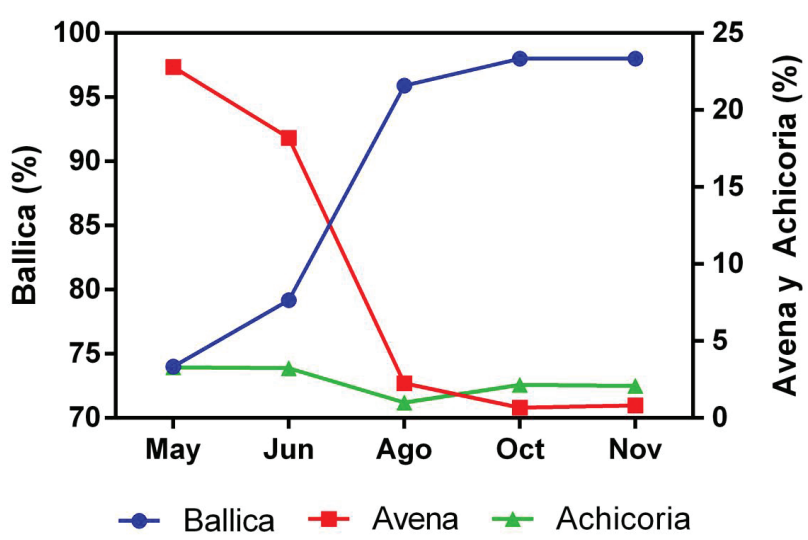

Figura 1. Porcentajes de composición botánica de ballica, avena y achicoria, en cinco cortes.

Figure 1. Percentages of botanical composition of ryegrass, oats and chicory, in five cuts.

menta de un $96 \%$ a un $98 \%$, donde la avena disminuye hasta alcanzar cerca de un $0 \%$ y achicoria tan solo un $2 \%$. Para el último corte, es decir, al mes de diciembre, la distribución porcentual de especies se mantiene en valores similares al corte anterior, lo cual implica que la ballica ha logrado ocupar casi un $100 \%$ en la composición botánica de las especies muestreadas, restringiéndose la especie achicoria a tan solo un $2 \%$.

Durante todo el período, ballica posee los valores porcentuales más altos, y la producción acumulada individual por encima de los otros componentes en estudio. La achicoria, por otro lado, no tiene gran impacto sobre la producción de la pradera, llegando sólo a los $165 \mathrm{~kg}$ durante la temporada, en comparación con ballica que logra $9548 \mathrm{~kg}$.

Por otra parte, puede que también haya afectado la emergencia de estas, lo que depende principalmente de la profundidad de siembra. En un estudio realizado por Sanderson y Elwinger (2000), obtienen como resultado que hay una gran variación del porcentaje de emergencia si la semilla está a 1 o a 6 centímetros de profundidad. Si la semilla se posiciona a $1 \mathrm{~cm}$, la emergencia es de un $60 \%$, mientras que si está a $6 \mathrm{~cm}$, el porcentaje de emergencia disminuye a un $27 \%$.

Los mismos autores, comparan la emergencia de dos cultivares de plantago con tres cultivares de achicoria, aquí se encuentran diferencias significativas entre especies, siendo el porcentaje de emergencia de plantago mucho mayor que el de achicoria, pero no se ven diferencias significativas entre cultivares.

\section{Producción por especie}

Según el Cuadro 1, la especie de mayor importancia durante el estudio fue ballica. Para poder analizar el
Cuadro 1. Producción (kg MS ha-1) por especies según fecha de corte.

Table 1. Yield (kg DM ha-1) by species cut-off date.

\begin{tabular}{lrrcc}
\hline \multirow{2}{*}{$\begin{array}{l}\text { Fecha, } \\
\text { Meses }\end{array}$} & \multicolumn{4}{c}{ Producción por especie (kg MS ha-1) } \\
\cline { 2 - 5 } & Avena & Ballica & Achicoria & Malezas \\
\hline Mayo & 190,2 & 613,4 & 24,8 & 2,7 \\
Junio & 103,8 & 442,6 & 15,1 & 1,4 \\
Agosto & 31,9 & 1433,6 & 18,4 & 5,0 \\
Octubre & 10,8 & 4288,5 & 57,0 & 9,6 \\
Noviembre & 0,00 & 2768,6 & 51,1 & 8,6 \\
\hline
\end{tabular}

desarrollo o participación de las especies en la pradera se analizó la distribución porcentual de cada especie en cada fecha de corte o muestreo

Al evaluar el establecimiento del cultivo según el Cuadro 1 los cultivares de $C$. intybus poseen valores muy bajos en comparación con gramíneas, lo cual confirma lo planteado por Ruiz (1996); Aedo (1996) y López (1996), quienes mencionan las características positivas de las ballicas y en especial de ballica italiana como especie pratense agresiva y rústica para su adaptación a condiciones edafoclimáticas de esta zona. Además Balocchi (2013), señala que un bajo poder de germinación afecta la composición botánica, en relación a aquellas semillas de mejor porcentaje germinativo, lo cual refrenda lo mencionado por Bavera (2009), al señalar que la mejor expresión de la achicoria ocurre cuando es establecida durante la primavera, por lo que en otoño tiene un peor establecimiento que en primavera, además de que tenía a la siembra otras dos especies de muy rápido establecimiento como lo son la avena y la ballica italiana, las que compitieron con la achicoria. Por este mismo motivo fue que el número de plantas fue tan bajo y la producción de materia seca fue pobre.

La producción de las mezclas forrajeras por especie durante el periodo del ensayo se presenta en las Figuras 2 y 3

Según las Figuras 1 y 2 la especie de mayor preponderancia en el ensayo es ballica. La Figura 2 muestra que existe poca importancia de la achicoria dentro de la producción de forraje de la pradera, además de que disminuye su importancia a medida que avanzan los cortes, lo cual confirma lo planteado por Ruiz (1996) y Aedo (1996), quienes mencionan ya sea el efecto de la temperatura en el crecimiento de gramineas y leguminosas y que las praderas del sur de Chile están compuestas principalmente por estas especies, lo que puede estar asociado a las condiciones edafoclimaticas ventajosas señalado por Peralta, (1976) y Ruiz (1996) quienes afirman que la ballica italiana por sus carac- 


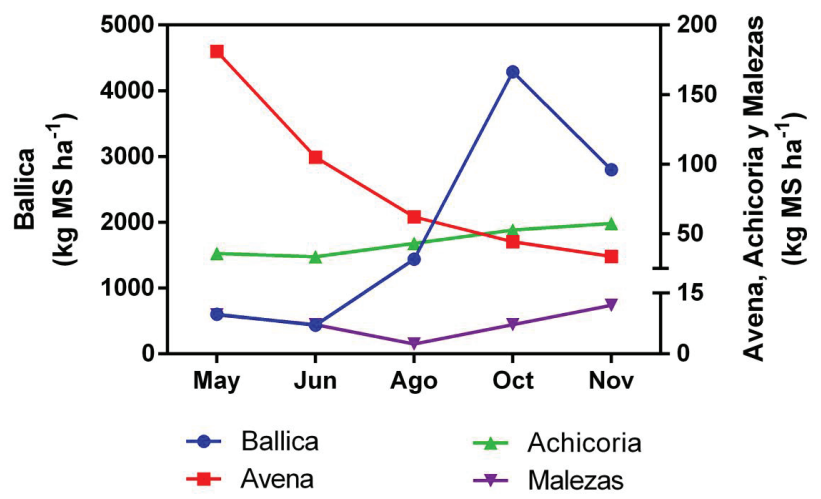

Figura 2. Producción (kg MS ha-1) por corte de ballica, avena, achicoria y malezas durante el periodo del ensayo.

Figure 2. Yield (kg DM ha-1) per cut of ryegrass, oats, chicory and weeds during the trial period.

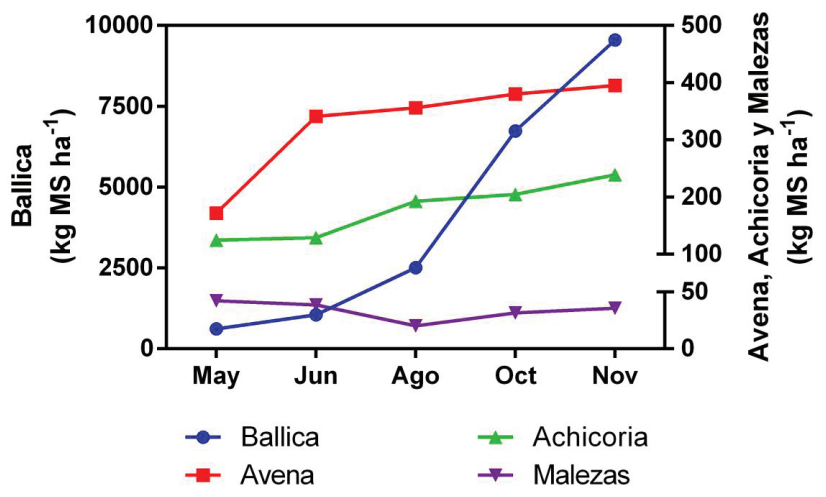

Figura 3. Producción acumulada (kg MS ha-1) según especies durante el periodo del ensayo.

Figure 3. Cumulated yield (kg DM ha-1) by species during the trial period.

terísticas morfológicas es mucho más agresiva en la ocupación del espacio que otras especies. Deak et al. (2007) corroboran lo anterior afirmando que la achicoria forrajera disminuye su contribución al aumentar el número de especies presentes.

No obstante lo anterior, los datos contrastan con los entregados por AGRISEEDS (2014) y Li y Kemp (2005) que indican que la especie $C$. intybus, como planta posee una persistencia relativamente buena al ser sembrada en mezcla con ballica perenne y trebol blanco, logrando un $22 \%$, de contribución en una producción total de la pradera de 16,2 ton MS ha-1 el año 1, valores superiores al ensayo, lo cual puede deberse entre otras causas a lo mencionado por Peralta (1976) y a lo señalado por Ruiz (1996) sobre la diferencia entre ballicas, pues el ensayo contempló mezcla de tres especies, entre ellas, ballica italiana, otra gramínea como avena y no trébol blanco como lo cita Li y Kemp (2005) y Bavera (2009), quienes recomiendan su siembra en mezcla con trébol blanco por su alta demanda de nitrógeno aportado por la leguminosa.

Bavera (2009), plantea que achicoria forrajera posee su mejor expresión en clima mediterráneo. Estero (2013), quien evaluó variedades de achicoria en Uruguay confirma lo mencionado por FONTAGRO (2011), en donde mediciones de establecimiento son superiores al ensayo, con $3147 \mathrm{~kg} \mathrm{MS} \mathrm{ha}^{-1}$, pero en Cochabamba, agro-clima y sitio edafoclimático no comparables con el sitio y condiciones del ensayo.

Bavera (2009) recomienda la defoliación con un mínimo de siete hojas y a una altura de $25-35 \mathrm{~cm}$, para que su sistema radical pueda crecer. En este ensayo se respetaron estas recomendaciones técnicas realizando los cortes a calendario fijo. Esto implica que las fechas de corte establecidas no serían las más indicadas para esta especie en esta zona agro-edafoclimática, para poder evaluar su producción en $\mathrm{kg} \mathrm{MS} \mathrm{ha-1.}$

Al cortar la pradera en invierno y al sumar a esto un mínimo crecimiento de la achicoria en invierno, se defoliaron las plantas con un menor número de hojas de lo recomendado, lo cual trajo consigo una mayor mortalidad de plantas en invierno y por ende un menor crecimiento en primavera de las plantas sobrevivientes. Esto afectó la composición botánica de la pradera. Por lo tanto, se concluye que, las fechas de corte, las condiciones de tamaño de semilla y agroclimáticas-edáficas no son ventajosas para la especie $C$. intybus, al ser sembradas con gramíneas. Se debe evaluar su siembra en mezcla con especies leguminosas para observar su establecimiento en comparación con los datos aportados por este estudio en fechas más oportunas o de mayor desarrollo foliar/radical.

\section{Análisis por cultivar de $C$. intybus}

Los resultados obtenidos en la producción acumulada de los cv, según Cuadro 2, muestran que se ubica primero Punter con $178 \mathrm{~kg} \mathrm{MS} \mathrm{ha}^{-1}$ producida desde la siembra hasta el quinto corte, seguido por Choice con una producción de 110,8 kg y la menos productora nuevamente fue Chicory 501 con un total producido de $64,1 \mathrm{~kg}$. Este último valor estaría en desacuerdo con lo aportado por AGRISEEDS (2014), en donde se señala que cv Chicory 501 por ser un cultivar de última generación poseería elevados rendimientos de $\mathrm{MS} \mathrm{ha}^{-1}$, lo cual implicaría que este cultivar, evaluado en las condiciones edafoclimáticas del estudio, no muestra similitud con lo mencionado por estos autores.

El cultivar SF Punter según Seed Force (2009) posee un gran vigor de establecimiento con buenas producciones de verano e invierno, lo que concordaría con los autores ya que la producción acumulada fue tres veces mayor a Chicory. El cultivar Choice, de producción in- 
termedia, que produjo casi el doble de Chicory 501, está de acuerdo con lo planteado por AGRICOM (2012), en cuanto a su alta productividad.

La Figura 4 muestra la producción acumulada y por corte de los tres cultivares a evaluar en su establecimiento.

De acuerdo con Cuadro 3, no existen diferencias significativas $(p>0,05)$ entre los tres cultivares durante las tres primeras fechas de corte (mayo, junio y agosto), pero si existen diferencias significativas para los siguientes meses (octubre y noviembre).

Las diferencias entre los cultivares indica que SF Punter muestra los mayores valores con 97,6 y $106 \mathrm{~kg}$ MS ha-1 para los meses de octubre y noviembre respectivamente, seguido por cv Choice con 56,3 y $40,5 \mathrm{~kg}$ MS ha ${ }^{-1}$ y finalmente Chicory 501 con 17,2 kg MS ha-1 disminuyendo a su valor más bajo durante el ensayo con tan solo 6,9 $\mathrm{kg} \mathrm{MS} \mathrm{ha-1}^{-1}$. La escala de valores de producción estaría de acuerdo a planteado por AGRICOM

Cuadro 2. Producción acumulada (kg MS ha-1) por cultivar en las distintas fechas de corte.

Table 2. Cumulated yield ( $\left.\mathrm{kg} \mathrm{DM} \mathrm{ha} \mathrm{a}^{-1}\right)$ per cultivar at different cutting dates.

\begin{tabular}{lccccc}
\hline \multicolumn{5}{c}{ Mes de Corte } \\
\hline Cultivar & Mayo & Junio & Agosto & Octubre & Noviembre \\
\hline \multicolumn{5}{c}{$\cdots \mathrm{kg} \mathrm{MS} \mathrm{ha}^{-1} \ldots$} \\
\hline Chicory 501 & 24,3 & 33,6 & 44,5 & 57,8 & 64,1 \\
Choice & 24,0 & 36,6 & 50,7 & 85,9 & 110,8 \\
Punter & 30,3 & 51,4 & 65,5 & 126,9 & 178,0 \\
\hline
\end{tabular}

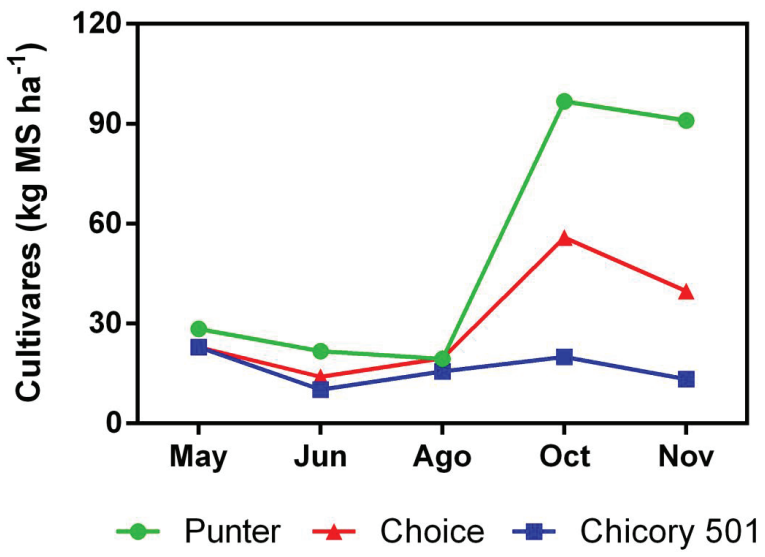

Figura 4. Producción (kg MS ha-1) por cultivar por fecha de corte.

Figure 4. Yield (kg DM ha-1) per cultivar per cut-off date.
Cuadro 3. Interacción cultivar mes de muestreo para la producción de materia seca por corte.

Table 3. Cultivar and sampling month interaction for dry matter yield per cut.

\begin{tabular}{|c|c|c|c|c|c|}
\hline Cultivar & Mayo & Junio & Agosto & Octubre & Noviembre \\
\hline & \multicolumn{5}{|c|}{$\cdots$ kg MS ha ${ }^{-1} \cdots$} \\
\hline Chicory 501 & $22,8 \mathrm{a}$ & $10,3 \mathrm{a}$ & $16,3 \mathrm{a}$ & $17,2 \mathrm{c}$ & $6,9 \mathrm{c}$ \\
\hline Choice & $23,1 \mathrm{a}$ & $13,4 \mathrm{a}$ & $19,4 \mathrm{a}$ & $56,3 \mathrm{~b}$ & $40,5 \mathrm{~b}$ \\
\hline Punter & $28,5 \mathrm{a}$ & $21,8 \mathrm{a}$ & $19,5 \mathrm{a}$ & 97,6 a & $106,1 \mathrm{a}$ \\
\hline
\end{tabular}

*Letras diferentes dentro de las columnas denotan que son estadísticamente diferentes $(\mathrm{p}<0,05)$.

(2012) y AGRISEEDS (2014) en cuanto a la potencia productiva de SF Punter y Choice pero no con lo planteado por el primer autor en cuanto al potencial de la variedad Chicory 501.

AGRISEEDS (2014) señala que en una escala de 0 a 9 , en la que 0 es la planta menos persistente y 9 la con mayor persistencia, la variedad de achicoria forrajera SF Punter obtiene un 7,7 siendo la mejor catalogada, mientras que despues le siguen Chicory 501 con un 7,0 y Choice con 4,7 puntos, además indica que sí hay diferencias significativas entre los distintos cultivares. Esto último se puede deber o tener causalidad en que la fecha de establecimiento para la variedad Chicory 501 no sea la indicada para este agroclima y que la especie achicoria es muy bajo su aporte en comparación con ballica y avena.

Las diferencias estadísticas entre los cultivares pueden deberse entre otros factores o causas a su diferente capacidad de crecimiento, por ende, genética, donde el cv. SF Punter en este ensayo muestra una mayor capacidad de producir materia seca acumulada, con 54,72 $\mathrm{kg}$ MS ha-1 por corte, seguido de Choice, que produjo $30,52 \mathrm{~kg} \mathrm{MS} \mathrm{ha}^{-1}$ promedio por corte y por último Chicory 501, la cual tuvo una producción de solo $14,71 \mathrm{~kg}$ MS ha ${ }^{-1}$ como promedio entre cortes.

\section{Composición morfológica}

En el Cuadro 4 se muestran los componentes morfológicos de las distintas variedades, se puede apreciar que no hay diferencias significativas $(p>0,05)$ en ninguno de los componentes en estudio. Además, se puede observar que, en el momento del corte para componentes morfológicos, las plantas estaban pequeñas, esto porque el número de hojas fue menor de tres.

Según Bavera (2009), se recomienda su corte con un mínimo de siete hojas, mientras que Lee et al. (2015a) recomiendan defoliaciones a una altura residual de entre 2,5 y $5,5 \mathrm{~cm}$ para no comprometer el 
rendimiento y entre 1,5 y 2,5 cm para no afectar negativamente la composición nutricional. Adicionalmente, Lee et al. (2015b) recomiendan al menos 21 días de rebrote para que la planta logre acumular una suficiente cantidad de carbohidratos de reserva. Al comparar los componentes morfológicos entre variedades obtuvimos que Choice es la variedad que produce las hojas de mayor tamaño, Chicory 501 la que produce más hojas, pero la que presentó mejor relación hoja/tallo según el peso de estos fue Punter. Dentro de todos estos análisis no hubo diferencias significativas, por lo que estas diferencias son mínimas y no debiesen producir diferencias en calidad u otros aspectos.

\section{Análisis nutricional por cultivar}

En el Cuadro 5 se muestra el análisis nutricional de cada una de las variedades de Achicoria evaluadas en el ensayo, éstas en general obtuvieron un alto contenido de energía metabolizable. Además, tiene bajos contenidos de fibra detergente neutro y ácido y un bajo extracto etéreo.

El mayor contenido de proteína bruta lo obtuvo la variedad Choice con un 27,24\%, seguida por Punter con $25,51 \%$ y por último Chicory 501 con $23,74 \%$.

En el contenido de proteina cruda, nuestro análisis entrega mejores resultados, presentando cerca de un
$25 \%$ vs. un $20 \%$ de Glassey et al. (2013), lo cual nos permite sugerir que los datos de Glassey et al. (2013), tienen que ser de plantas más maduras, o que su análisis fue realizado en una etapa mas tardía del cultivo. Sin embargo, al compararlos en energía metabolizable, los datos son similares a los aportados por Sepúlveda et al. (2011). Lo que implica que la energía es un parámetro similar pues los ensayos fueron realizados en similar época.

El cv que presenta una menor energía es Punter, con

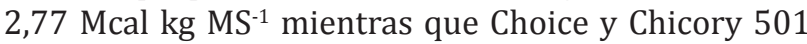
son similares con 2,82 $\mathrm{Mcal}_{\mathrm{kg} \mathrm{MS}}^{-1}$.

Al referirnos al contenido de cenizas totales de los cv en estudio, no existen marcadas diferencias, puesto que al compararlos con Sepúlveda et al. (2011), las diferencias son de 5\%. No obstante Glassey et al. (2013) entregan en su estudio contenidos de cenizas totales cercanas al $14 \%$, lo que refleja diferencias de $2 \%$ con el contenido de cenizas totales (12\%) obtenidas en este estudio.

Los datos del estudio para FDN son distintos en relación a los aportados por Sepúlveda et al. (2011). Los cv de achicoria presentan contenidos de fibra detergente acido y neutro mucho menores que la pradera permanente. Es así como la FDN de la pradera es 38,4\%, cv Choice un 25,19\%, Chicory 501 un 28,32\% y Punter $27,44 \%$, lo que nuevamente sugiere la diferencia entre las especies en las mediciones según paráme-

Cuadro 4. Componentes morfológicos de cada variedad.

Table 4. Morphological components of each variety.

\begin{tabular}{lcccc}
\hline Componente Morfológico & Chicory 501 & Choice & Punter & Significancia \\
\hline Largo Lámina (mm) & 12,62 & 13,38 & 12,23 & $\mathrm{~ns}$ \\
Número hojas & 2,98 & 2,50 & 2,46 & $\mathrm{~ns}$ \\
Peso hojas (g) & 0,32 & 0,46 & 0,54 & $\mathrm{~ns}$ \\
Peso peciolos (g) & 0,18 & 0,18 & 0,22 & $\mathrm{~ns}$ \\
Relación hoja/peciolo & 1,83 & 2,40 & 2,70 & $\mathrm{~ns}$ \\
\hline
\end{tabular}

ns: No significativo.

Cuadro 5. Análisis nutricional según variedades de achicoria.

Table 5. Nutritional analysis according to varieties of chicory.

\begin{tabular}{lcccccccc}
\hline \multirow{2}{*}{ Cultivar } & MS & CT & PB & EE & FDN & FDA & VD & EM \\
\cline { 2 - 9 } & & & & $\cdots(\%) \cdots$ & & & & (Mcal kg MS $\mathbf{~ M S ~}^{-1}$ \\
\hline Chicory 501 & 8,7 & 11,9 & 23,7 & 4,3 & 25,2 & 20,1 & 78,1 & 2,82 \\
Choice & 8,9 & 11,6 & 27,2 & 3,8 & 28,3 & 21,4 & 78,4 & 2,82 \\
Punter & 9,1 & 12,9 & 25,5 & 4,3 & 27,4 & 20,6 & 76,5 & 2,77 \\
\hline
\end{tabular}

MS: materia seca; CT: cenizas totales; PB: proteína bruta; EE: extracto etéreo; EM: energía metabolizable; FDN: fibra detergente neutro; FDA: fibra detergente ácido; VD: digestibilidad verdadera. 
tro. Por otro lado, la FDA de la pradera del estudio fue de un 26\% y la FDA de las achicorias fueron de 20,1, 21,4 y $20,6 \%$ para Chicory 501, Choice y Punter respectivamente.

\section{CONCLUSIONES}

Al evaluar el establecimiento de los tres cv de $C$. intybus, el aporte productivo de los cultivares durante los primeros meses no se encontraron diferencias entre estos, siendo diferentes únicamente en los dos últimos meses (octubre y noviembre), siendo Punter el de mejor producción. Por lo tanto, la fecha de corte es un factor que influye en la expresión productiva de los cultivares, donde durante el invierno los tres se ven afectados por clima, suelo y competencia con otras especies de mayor agresividad.

En cuanto a la contribución de la especie $C$. intybus en la mezcla forrajera empleada, la composición botánica muestra que la especie con mayor contribución de la mezcla forrajera es ballica italiana, con un $98 \%$ acumulado al final del ensayo. De esta forma, no se recomienda el establecimiento de achicoria en mezcla con especies gramíneas de rápido establecimiento como ballica bianual y avena.

\section{REFERENCIAS}

Aedo, N., 1996. Morfología de una gramínea y leguminosa típica, in: Ruiz, I. (Ed.), Praderas para Chile, 2 ${ }^{\mathrm{a}}$ Ed. Instituto de Investigaciones Agropecuarias (INIA). Santiago, Chile, pp. 27-29. http://www2.inia.cl/medios/biblioteca/libros/NR30968.pdf

AGRICOM, 2012. Choice chicory. http://www.agricom.com. au/products/herbs/chicory/choice-chicory (acceso, 08.04.2013).

AGRISEEDS, 2014. Pasture Cultivars. Pasture and Forage Manual. pp. 39-41.

Bavera, G., 2009. Achicoria. Sitio argentino de la producción animal. http://www.produccion-animal.com. ar/produccion_y_manejo_pasturas / pasturas $\% 20$ artificiales/120-achicoria.pdf (acceso, 27.05.2013)

Deak, A., Hall, M.H., Sanderson, M.A., Archibald, D.D., 2007. Production and Nutritive Value of Grazed Simple and Complex Forage Mixtures. Agronomy Journal 99(3), 814-821. http://doi:10.2134/agronj2006.0166

Estero, 2013. Achicoria Salad A. Forrajera de muy alta producción y persistencia. http://www.estero.com.uy/ images/fichas_en_PDF/pasturas_fichas/ficha_de_pasturas_otras/Achicoria\%20Salad\%20A.pdf (acceso, 12.05.2013).

FONTAGRO, 2011. VI Taller de seguimiento técnico de proyectos FONTAGRO. Centro de Investigación en Forrajes "La Violeta". Universidad Mayor de San Simón. Cochabamba, Bolivia. https://www.fontagro.org/wp-con-
tent/uploads/2005/01/pp_ISTA_05_787_2011_bolivia. pdf (acceso, 12.05.2013).

Glassey, C.B., Clark, C.E.F., Roach, C.G., Lee, J.M., 2013. Herbicide application and direct drilling improves establishment and yield of chicory and plantain. Grass and Forage Science 68(1), 178-185. https://doi.org/10.1111/ j.1365-2494.2012.00885.x

Lee, J.M., Hemmingson, N.R., Minnee, E.M.K., Clark, C.E.F., 2015a. Management strategies for chicory (Cichorium intybus) and plantain (Plantago lanceolata): Impact on dry matter yield, nutritive characteristics and plant density. Crop and Pasture Science 66(2), 168-183. https:// doi.org/10.1071/CP14181

Lee, J.M., Minnee, E.M.K., Clark, C.E.F., 2015b. Patterns in non-structural carbohydrate and nitrogen reserves in chicory (Cichorium intybus L.) and plantain (Plantago lanceolata L.) during regrowth in summer. Crop and Pasture Science 66(10), 1071-1078. https://doi. org/10.1071/CP15122

Li, G., Kemp, P., 2005. Forage Chicory (Cichorium intybus L.): A review of its agronomy and animal production. Advances in Agronomy 88, 187-222. https://doi.org/10.1016/ S0065-2113(05)88005-8

López, H., 1996. Especies Forrajeras Mejoradas, en: Ruiz, I. (Ed.), Praderas Para Chile 2 ${ }^{\underline{a}}$ Ed. Instituto de Investigaciones Agropecuarias (INIA), Santiago, pp. 41108. http://www2.inia.cl/medios/biblioteca/libros/ NR30969.pdf

Peralta, M., 1976. Uso, clasificación y conservación de suelos. Servicio Agrícola y Ganadero. Santiago, Chile.

Reed, K.F.M., Nie, Z.N., Miller, S., Hackney, B.F., Boschma, S.P., Mitchell, M.L., Albertsen, T.O., Moore, G.A., Clark, S.G., Craig, A.D., Kearney, G., LI, G.D., Dear, B.S., 2008. Field evaluation of perennial grasses and herbs in southern Australia. 1. Establishment and herbage production. Australian Journal of Experimental Agriculture 48(4), 409-423. http://doi.org/10.1071/EA07135

Ruiz, I., 1996. La pradera en el llano longitudinal y valles de la zona central, in: Ruiz, I. (Ed.), Praderas para Chile, $2^{\text {a }}$ Ed. Instituto de Investigaciones Agropecuarias (INIA). Santiago, Chile, pp. 495-504. http://www2.inia.cl/medios/ biblioteca/libros/NR30990.pdf

Rumball, B., 1986. Grasslands Puna' chicory (Cichorium intybus L.), New Zealand Journal of Experimental Agriculture 14(1), 105-107, http://doi.org/10.1080/0301552 1.1986.10426133

Sanderson, M., Elwinger, G.F., 2000. Chicory and English Plantain Seedling Emergence at Different Planting Depths. Agronomy Journal 92, 1208- 1209. http://doi. org/10.2134/agronj2000.9261206x

Seed Force, 2009. Chicory SF Punter. http://www.seedforce. com.au/userfiles/files/SF_Punter.PDF (08.04.2013).

Sepúlveda, P., Wittwer, F., Bohmwald, H., Pulido, R., Noro, M., 2011. pH ruminal y balance metabólico de $\mathrm{Mg}$ en vacas lecheras en pastoreo suplementadas con oxido de magnesio. Archivos de Medicina Veterinaria 43(3), 241-250. http://dx.doi.org/10.4067/S0301732X2011000300006 
